\title{
Compute-and-Forward with Relay Selection: A Cooperative Game
}

\author{
Koralia N. Pappi*, Panagiotis D. Diamantoulakis*, and George K. Karagiannidis*† \\ *Department of Electrical and Computer Engineering, Aristotle University of Thessaloniki, GR-54124 Thessaloniki, Greece \\ ${ }^{\dagger}$ Department of Electrical and Computer Engineering, Khalifa University, PO Box 127788, Abu Dhabi, UAE \\ e-mails: \{kpappi,padiaman\}@auth.gr, geokarag@ieee.org
}

\begin{abstract}
Motivated by the cooperative game theory, we propose an efficient physical layer network coding technique for compute-and-forward $(\mathrm{C \& F})$ relaying systems, which jointly optimizes the sum and the minimum transmission rate, while minimizing the total transmitting power. Specifically, we show that relay selection in C\&F networks leads to an increase of both rates. We also propose an algorithmic approach for the rates' further optimization and we illustrate that its implementation can offer the same or even greater gain than adding an extra relay. Furthermore, the tradeoff between the minimum and the sum rate is explained by utilizing the concept of Pareto frontier. Simulations and numerical results show that a combination of relay selection and the proposed algorithms offers a considerable increase in the sum rate for the same transmitting power, without reducing the system's Quality of Service.
\end{abstract}

\section{INTRODUCTION}

Compute-and-Forward $(\mathrm{C} \& \mathrm{~F})$, first introduced in the pioneering work of B. Nazer and M. Gastpar [1], is a promising technique which exploits interference in multiuser wireless networks in order to achieve high transmission rates. A C\&F relay decodes an integer equation of the transmitted coded messages, with the use of nested lattice codes [2]. The decoded equation is then forwarded towards a Centralized Decoder (CD) which, having enough independent equations, decodes the transmitted messages from all users. The choice of integer equation coefficients at each relay is performed with the use of Physical Layer Network Coding (PNC), first introduced in [3] as a special case of Network Coding (NC) [4], [5].

Game theory provides a formal analytical framework with a set of mathematical tools to study the complex interactions among rational players. Recently, game theory has emerged as a tool for communication network analysis and specifically network coding [6]. The choice of integer equation coefficients by a $\mathrm{C} \& \mathrm{~F}$ relay is an optimization problem which has no analytical solution. Various works have proposed algorithmic solutions to this problem, e.g. using Lenstra-Lenstra-Lovász (LLL) lattice reduction [7], or a modified Fincke-Pohst method [8], among others. However, when various C\&F relays forward their equations to a $\mathrm{CD}$, the set of equations must be independent, which may not be achieved if each relay independently

The work of G. K. Karagiannidis is funded by the KHALIFA University, under the 2014 KUIRF-Level 1 project: Revisiting Fundamental Concepts for Green Wireless Communications Networks (REFORM). chooses its integer equation coefficients. Thus, the available relays need to cooperate in order to select the best possible independent set of equations and they can be considered as players in a cooperative game. Furthermore, the achievable transmission rates depend on the combination of equations and not on each one individually. Thus, the relays need to cooperate in order to jointly optimize the PNC process.

In [8], the authors propose an optimization of the equation coefficient vectors so that the relays provide the $\mathrm{CD}$ with a set of independent equations, based on the maximization of the minimum computation rate. Similar strategy was followed in [9] for Complete-C\&F and Incomplete-C\&F. However, both approaches do not deal with the sum rate, which is an important metric of the network performance, since in most network scenarios, users do not have the same needs for transmission rate and Quality of Service (QoS) requirements. Furthermore, in a C\&F network with $L$ sources and $M$ relays, where $M \geq L$, only $L$ relays are needed. Relay selection for a cloud base station is investigated for the first time in [10], where the relays choose equation coefficients independently and an exhaustive search for the best combination is performed. However, although this solution is useful, it is suboptimal, since the relays always choose one specific coefficient vector each, beforehand. Thus, no cooperation or tradeoff between relays for the rates' optimization can be performed, while an independent set of equations is not always guaranteed.

In this work, a $C \& F$ network employing surplus relays with respect to the number of the sources is considered. An efficient method for the joint optimization of the sum and the minimum rate and the best selection of relays through the PNC is proposed, where the relays cooperate in order to form the best coalition, which will forward equations towards the CD. Thus, the relay selection is matched to a gametheoretical coalition formulation problem. The formation of the best coalition is performed with respect to one of the following criteria: (i) the maximization of the minimum transmission rate over the network and (ii) the maximization of the sum rate under minimum transmission rate constraints for each source. The Pareto-optimal frontier is used to define the dominating solutions and to show the tradeoff between the maximization of the minimum and the sum rate.

Extensive simulations have been conducted to evaluate the 
performance of the proposed selection algorithms. It is remarkable that algorithmic optimization may be more beneficial compared to adding more relays to the network. In fact, when both strategies are combined, our method maximizes the sum rate, under predetermined thresholds on the minimum rate and the transmitting power. The proposed strategy can be efficiently used in the uplink of cloud base stations, making it a promising candidate for future networks, such as the Cloud Radio Access Network (CRAN) [11].

\section{SySTEM MODEL}

\section{A. Compute-and-Forward Network}

We consider a real-valued C\&F network as in [1] with $L$ sources, denoted by $S_{l}, l=1,2, \ldots, L, M$ relays, denoted by $R_{m}, m=1,2, \ldots, M$, where $M \geq L$, and a $\mathrm{CD}$, which is connected to the relays via error free bit pipes. The channel matrix $\mathbf{H}$ is given by

$$
\mathbf{H}=\left[\mathbf{h}_{1}, \mathbf{h}_{2}, \ldots, \mathbf{h}_{M}\right], \quad \mathbf{h}_{m}=\left[h_{m, 1}, h_{m, 2}, \ldots, h_{m, L}\right]^{T}
$$

where $\mathbf{h}_{m}$ is the channel coefficient vector of length $L$ as seen by the relay $R_{m}, h_{m, l}$ is the real channel coefficient between the source $S_{l}$ and the relay $R_{m}$, and [.] ${ }^{T}$ denotes transposition.

Each relay receives a signal

$$
\mathbf{y}_{m}=\sum_{l=1}^{L} h_{m, l} \mathbf{x}_{l}+\mathbf{z}_{m}
$$

where $\mathrm{x}_{l} \in \mathbb{R}^{N}$ is the signal vector transmitted by the source $S_{l}, \mathbf{y}_{m} \in \mathbb{R}^{N}$ is the received vector by the relay $R_{m}, N$ is the dimension of the nested lattice code [2] used in the C\&F scheme and $\mathbf{z}_{m} \in \mathbb{R}^{N}$ is the noise vector which follows a normal distribution $\mathcal{N}\left(\mathbf{0}, \mathbf{I}_{N \times N}\right)$, with $\mathbf{0}$ being the zero vector of length $N$ and $\mathbf{I}_{N \times N}$ being the $N \times N$ unitary matrix. Each transmitted signal is subject to a power constraint $P$, i.e. $\frac{1}{N}\left\|\mathbf{x}_{l}\right\|^{2} \leq P$, where $\|\cdot\|$ denotes the vector norm.

The relays then decode an integer equation of the transmitted vectors, with equation coefficients given by the vectors

$$
\mathbf{a}_{m}=\left[a_{m, 1}, a_{m, 2}, \ldots, a_{m, L}\right]^{T} .
$$

Thus, each relay decodes a codeword

$$
\widehat{\mathbf{x}}_{m}=\left[\sum_{l=1}^{L} a_{m, l} \mathbf{x}_{l}\right] \bmod \Lambda
$$

where $[\cdot] \bmod \Lambda$ is the modulo lattice operation on the coarse lattice of the nested lattice code in use [1], [2].

\section{B. Compute-and-Forward Rates}

The choice of the equation coefficient vector determines the rate that a relay can achieve, while decoding equations of messages. It was proven in [1] that the achievable computation rate region of a relay $R_{m}$ for a given choice of $\mathbf{a}_{m}$ and given channel coefficients is

$$
\mathcal{R}_{m}^{r}=\frac{1}{2} \log _{2}^{+}\left(\left\|\mathbf{a}_{m}\right\|^{2}-\frac{P\left(\mathbf{h}_{m}^{T} \mathbf{a}_{m}\right)^{2}}{1+P\left\|\mathbf{h}_{m}\right\|^{2}}\right)^{-1}
$$

where $\log _{2}^{+}(\cdot)=\max \left(\log _{2}(\cdot), 0\right)$ and $(\cdot)^{r}$ denotes the computation rate of a relay. The maximization of the rate in (5) with respect to the equation vector $\mathbf{a}_{m}$ is achieved when [7]

$$
\mathbf{a}_{m}=\arg \min _{\mathbf{a} \in \mathbb{Z}^{L}, \mathbf{a} \neq 0} \mathbf{a}^{T} \mathbf{G a}
$$

where $\mathbf{G}=\mathbf{I}_{L \times L}-\frac{P \mathbf{h}_{m} \mathbf{h}_{m}^{T}}{1+P\left\|\mathbf{h}_{m}\right\|^{2}}$.

As mentioned before, the $\mathrm{CD}$ can decode all messages if it is provided with $L$ independent equations. Thus, not all $M$ relays are needed to operate at all times, but a subset of $L$ out of $M$ relays. The CD needs to invert the $L \times L$ full rank network coding matrix $\mathbf{A}$, whose columns are the equation coefficient vectors $\mathbf{a}_{m}$ of the $L$ selected relays:

$$
\mathbf{A}=\left[\mathbf{a}_{m 1}, \mathbf{a}_{m 2}, \ldots \mathbf{a}_{m L}\right]
$$

where $m 1, m 2, \ldots, m L$ are the indices of the $L$ selected relays. We consider $\mathbf{a}_{m}=\mathbf{0}$ for the relays which are not selected. The transmission rate of a given source is bounded by the minimum computation rate among only those relays which decode an equation containing the codeword transmitted by this source. That is [1],

$$
\mathcal{R}_{l}^{s} \leq \min _{a_{m, l} \neq 0} \mathcal{R}_{m}^{r}
$$

where $(\cdot)^{s}$ denotes a transmission rate from a source.

Remark 1: Each source can transmit with different rate [1], as shown by (8), where the $l$-th transmission rate is defined only by the equation coefficient vectors with non-zero elements at the $l$-th position. Thus a vector a $\in \mathbf{A}$ which achieves the minimum computation rate, defines only those transmission rates corresponding to its non-zero elements.

Remark 2: The maximization of the sum rate may be conflicting to the maximization of the minimum transmission rate over the network. According to Remark 1, it may be favorable for the minimum rate to be defined by a vector a which achieves lower rate but contains many zero elements. In this case, the sum rate may increase, since the transmission rates corresponding to the zero elements of a will be defined by other vectors with higher computation rates.

\section{PHY-Layer Network Coding Optimization Criteria}

In this work, we consider two criteria, under which the optimization of the PNC will be performed.

1) Maximization of the minimum transmission rate: This criterion ensures that the source, which is affected the most by the channel conditions, will be allowed to transmit at the highest rate possible. The minimum transmission rate in the network also coincides with the minimum computation rate at the selected relays. If $\mathcal{M}$ is the set with cardinality $M$, containing all available relays, and $\mathcal{M}^{\prime}$ is a set of cardinality $L$, containing $L$ out of $M$ relays, then for the network coding matrix A holds that

$$
\mathbf{A}=\arg \max _{|\mathbf{A}| \neq 0, \mathcal{M}^{\prime} \subseteq \mathcal{M}}\left(\min _{m \in \mathcal{M}^{\prime}}\left(\mathcal{R}_{m}^{r}\right)\right) .
$$

The criterion in (9) was partly dealt with in [8], for the case of $L=M$, that is, for the case of no relay selection. Also note 
that (9) may have more than one solutions for the network coding matrix A.

2) Maximization of the sum rate under specifications for the minimum transmission rate: In some networks, the requirements of each user in terms of rate are different (e.g. they use different services etc.). Thus, an optimal strategy for such a network may be the maximization of the sum rate under the fulfilment of minimum requirements for each user, as long as at least one is at most equal to the maximum minimum rate (in the opposite case, the requirements cannot be satisfied simultaneously). This criterion can be formulated as follows.

$$
\begin{array}{ll}
\text { find } & \mathbf{A}=\arg \max _{|\mathbf{A}| \neq 0}\left(\sum_{l=1}^{L} \mathcal{R}_{l}^{s}\right) \\
\text { subject to } & \mathcal{R}_{l}^{s} \geq V_{l}, \quad l=1,2, \ldots, L
\end{array}
$$

where $V_{l}$ is the minimum rate for the source $S_{l}$.

\section{PNC As a CoAlitional Game}

\section{A. Essential Elements and Coalitions of the Game}

Below we define the essential elements of the game described briefly in Table I [6].

Definition 1: The $M$ available relays of the network are considered to be the players of the game. At each decision point, they have knowledge of all candidate vectors and the corresponding computation rates of the other players, while they know which of them is (or is not) already in a coalition. The above constitute the information available at each decision point. The actions available to each player at each decision point are whether they will enter a coalition or not, and which candidate equation coefficient vector they will choose. Finally, since the game is cooperative, instead of individual payoffs, the players try to maximize a total utility under various constraints, depending on the optimization criterion. The total utility is either the minimum rate or the sum rate of the network.

Relay selection can be described from a game theoretical perspective as a coalition formation among players. The game is then characterized as coalition formation game [6]. In the case where $L=M$, all relays must operate, and then the game is a canonical coalitional game.

Definition 2: The coalition of the game consists of the relays which are selected to forward equations to the $\mathrm{CD}$. When $L=M$, all relays participate in the coalition, which is a grand coalition.

\section{B. The Core of the Game}

At each decision point of the Game, as it will be described in Section IV, a new coalition and a new coding matrix $\mathbf{A}$ are formed, only if this leads to an increased total utility, under the constraint that the matrix $\mathbf{A}$ is full rank. Note that, the core is a solution concept in coalitional games that characterizes how the players form coalitions. The core is an achievable value of the total utility, for which no coalition can achieve a greater value. The existence of the core guarantees that there is no profitable coalitional deviation.

Theorem 1: The core of the game is non-empty, when among all possible combinations of selected vectors there is at least one, which leads to a full rank matrix $\mathbf{A}$.
TABLE I

Network Coding Game Among Relays

\begin{tabular}{l|l|l}
\hline & Minimum Rate & Sum Rate \\
\hline \hline Players & \multicolumn{2}{|l}{ Relays in set $M$} \\
\hline Strategies & \multicolumn{2}{|l}{$\begin{array}{l}\text { Each relay selects its network coding vector to guarantee } \\
\text { full rank A and maximize the total utility. }\end{array}$} \\
\hline \multirow{2}{*}{ Total utility } & $\min _{m} \mathcal{R}_{m}^{r}$ & $\sum_{l=1}^{L} \mathcal{R}_{l}^{s}$ \\
\hline Class & $\begin{array}{l}\mathbf{M}=\mathbf{L}: \text { Canonical Coalitional Game } \\
\mathbf{M}>\mathbf{L}: \text { Coalition Formation Game }\end{array}$ \\
\hline
\end{tabular}

Proof: When $L=M$, it is evident that for each relay $m \in$ $\mathcal{M}$ the marginal contribution of this relay, i.e. the difference between the value of a coalition with and without this relay, is non-decreasing, since a selfish choice of each player may lead to a coding matrix $\mathbf{A}$ which is not full rank, or for the case of the sum rate total utility, it may lead to lower values, depending on the zero elements of the solution. Therefore, the convexity property is satisfied and the core of the game is nonempty [6]. When $M>L$, the core of the game is non-empty since among the $M$ relays, there will always be at least one set $\mathcal{M}^{\prime}$ of $L$ relays that can jointly act better, by increasing the total utility. Hence these $L$ relays form the top coalition (and thus a weak top coalition as well). Therefore, the weak top-coalition property is satisfied and the coalition structure $\mathcal{M}^{\prime}$ is a core partition [12].

\section{The Pareto Frontier}

Often the two utility functions of Table I are conflicted, as explained in Remark 2. To better explain and face this conflict, we utilize the concept of Pareto frontier, which, for this game, is defined as follows.

Definition 3: The Pareto Frontier is the set of matrices that are not strictly dominated by any other matrix. The matrix $\mathbf{A}^{\prime}$ is said to Pareto dominate another matrix $\mathbf{A}\left(\mathbf{A}^{\prime} \succ \mathbf{A}\right)$, if

$$
\min _{m: \mathbf{a}_{m} \in \mathbf{A}^{\prime}} \mathcal{R}_{m}^{r} \geq \min _{m: \mathbf{a}_{m} \in \mathbf{A}} \mathcal{R}_{m}^{r} \text { and } \sum_{\substack{l=1 \\ \mathbf{a}_{m} \in \mathbf{A}^{\prime}}}^{L} \mathcal{R}_{l}^{s} \geq \sum_{\substack{l=1 \\ \mathbf{a}_{m} \in \mathbf{A}}}^{L} \mathcal{R}_{l}^{s}
$$

when the equalities do not hold simultaneously.

Formally, this defines a partial order on all possible matrices $\mathbf{A}$, and the Pareto Frontier is the set of maximal elements with respect to this order.

\section{COALITION Formation AND TOTAL UTILITY MAXIMIZATION STRATEGY}

\section{A. Formation of the candidate equation coefficient vector sets}

When each relay chooses its equation coefficient vector selfishly according to the criterion in (6), the problem can be mapped to finding the shortest vector on a lattice with generator matrix G, as in [7]. Thus, various algorithms are used, which originate in applications of lattice theory. For example, in [8], the authors applied a modified Fincke-Pohst method [13], for finding the lattice points closer to the origin of the lattice, which also correspond to the equation coefficient 
vectors achieving the highest rate. However, the authors predetermine the length of the set of candidate coefficient vectors by experience/simulations, which may lead to an omission of the joint optimal solution.

In this work, we dynamically set the length of the candidate sets, in order for the game to have a non-empty core, as dictated by Theorem 1, that is, there must be at least one full rank matrix $\mathbf{A}$ in the solution set. This can be achieved as follows. Since a full rank matrix A spans the $L$-dimensional space, in order for a full rank matrix $\mathbf{A}$ to exist, it is sufficient if the candidate equation coefficient sets of at least $L$ out of $M$ players also span the $L$-dimensional space. Thus, in the case of criterion 1 , we construct the candidate equation coefficient vector sets $\Omega_{m}$, using the Fincke-Pohst algorithm, adding vectors until they span the $L$-dimensional space for the first time. The above formation of the candidate sets $\Omega_{m}$ is slightly modified, if the optimization is performed according to criterion 2. Then the Fincke-Pohst algorithm constructs the set with all those vectors which can achieve a computation rate satisfying the QoS requirements of the sources. That is, all vectors in $\Omega_{m}$ with non-zero element in the $l$-th position, must achieve rate at least equal to the minimum requirement of the $l$-th source. According to the candidate set $\Omega_{m}$, each relay also constructs the set of corresponding achievable computation rates, denoted by $\Gamma_{m}$, as in [8].

\section{B. Maximization of the minimum transmission rate}

At each decision point of the game, the players have the information of all achievable rates among all players, that is, the sets $\Gamma_{m}$. These are sorted in descending order in a list $Q$. Note that the information between players is exchanged through the $\mathrm{CD}$ via error free bit pipes, thus there is no need for wireless interaction between the relays. The players cooperate in order to form a coalition of $L$ relays, which maximizes the minimum transmission rate, or equivalently the minimum computation rate.

It is natural that the first set of players which try to form a coalition are those, whose best individual choice of rate is among the $L$ highest out of $M$ individual best rates. Thus the first player who enters the coalition is the $m$-th relay whose maximum element in $\Gamma_{m}$ is greater than exactly $M-L$ maximum elements of the sets $\Gamma_{m}$ of all other players. Those players which can achieve higher rate than this, try to form a full rank matrix $\mathbf{A}$ using their corresponding vectors. The same priority is kept among these players, that is, the second player who tries to enter the coalition is the one whose maximum element in $\Gamma_{m}$ is greater than exactly $M-L+1$ maximum elements of the rest of the players etc. If there is no possible solution, the coalition is deformed, and the player which enters first the new coalition is the one who achieves the next rate in the sorted list $Q$. This process is repeated recursively, until the first full rank matrix $\mathbf{A}$ is formed.

Note that this recursion, which is not necessarily performed in [8], is a prerequisite for the maximization of the sum rate performed in the next section. This strategy is formulated in
Algorithm 1, which is executed for $l=L$ and $\mathbf{A}_{l}$ denotes the $l$ columns of $\mathbf{A}$ achieving the lowest computation rates.

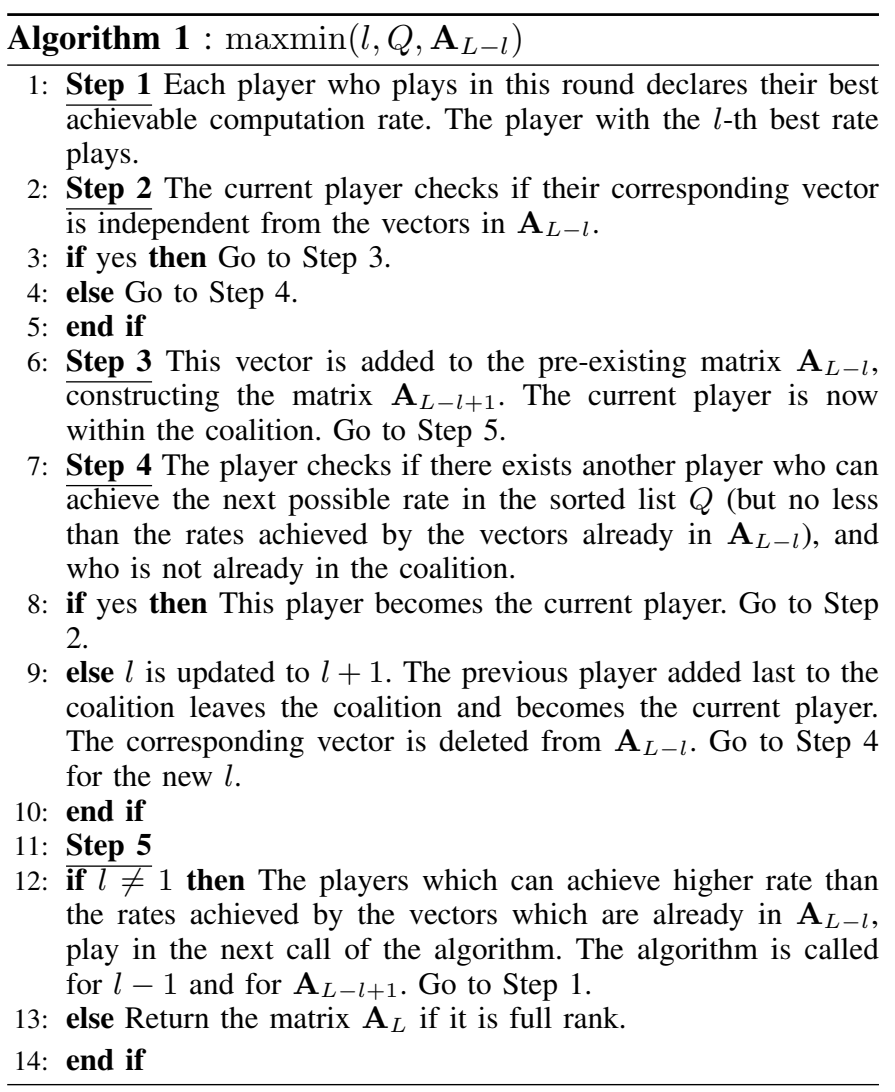

\section{Maximization of the sum rate}

The maximization of the sum rate utilizes Algorithm 1 which is first implemented, providing Algorithm 2 with a full rank matrix A. This algorithm is based on the observation in Remark 2. Thus, it permits each vector in the predetermined network coding matrix $\mathbf{A}$ to be replaced by a vector of lower computation rate, only if a weight for this vector is higher than the already achieved sum rate. This weight is an ideal sum rate that this vector can achieve. It is the sum of already determined transmission rates and of the best values for the undetermined transmission rates. The use of weights drastically reduces the possible combinations of vectors for a full rank matrix $\mathbf{A}$ and, there is no need to search all the elements of the sorted list $Q$.

The rest of the vectors in the new coding matrix are determined again applying Algorithm 1, to ensure that the newly formed matrix is full rank and to search if some vectors can achieve higher computation rates than before. This new formation is referred to as candidate coalition, because if its sum rate is lower than the previously achieved sum rate, it is rejected. Algorithm 2 is implemented recursively, from the vector of lower to the vector of higher computation rate in $\mathbf{A}$. Again, $\mathbf{A}_{l}$ denotes the $l$ columns of $\mathbf{A}$ achieving the lowest computation rates and refers to the candidate coalition, while matrix A corresponds to the best coalition formed up to the point of execution. The Algorithm is executed for $l=L$. 


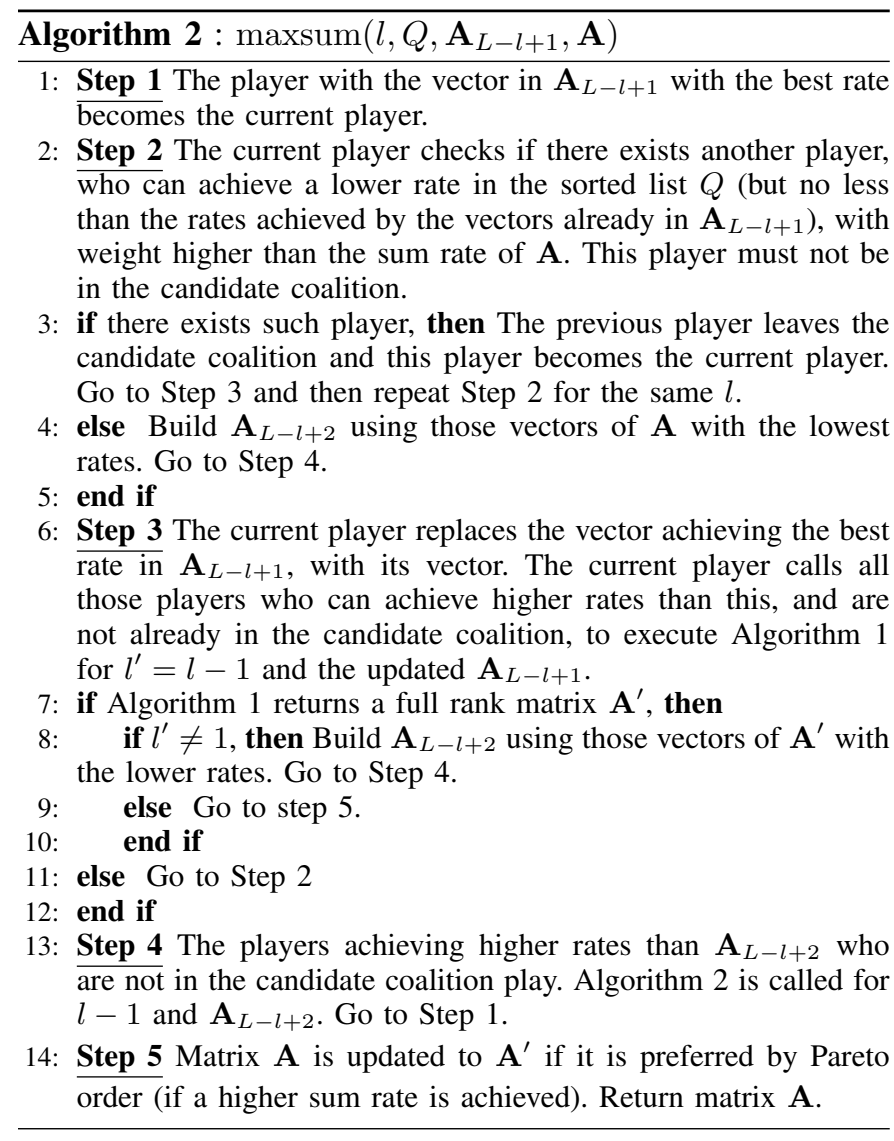

Note that the complexity of the algorithms is sufficiently low for real time execution, while the interaction among the players is finite and always less than an exhaustive search, since the sets $\boldsymbol{\Omega}_{m}$ are finite. Furthermore, note that the optimal solution is always included in the search space and is also the convergence point of the algorithms. For example, for a network with $L=4$ sources and $M=6$ relays, the construction of the vector sets and the implementation of Algorithms 1 and 2 for a specific channel realization are executed in MATLAB R2012b on a general purpose computer in 0.1 seconds.

\section{NUMERICAL RESULTS AND DISCUSSION}

\section{A. Pareto Frontier Example}

We present an example concerning a network with $L=4$ sources and $M=5$ relays, for a random channel instance. This example presents the solution set containing all possible full rank matrices $\mathbf{A}$, and the minimum and sum rate that each solution achieves.

In Fig. 1, the Pareto frontier consists of three distinct points, marked with asterisks. These solutions illustrate the tradeoff between the maximization of the minimum rate and the maximization of the sum rate. Note that, if Algorithm 1 is applied, the proposed strategy chooses as an optimal solution the one which corresponds to point Z. When Algorithm 2 is applied, with a minimum requirement of $\mathcal{R}_{m}^{r} \in[0,0.4047]$

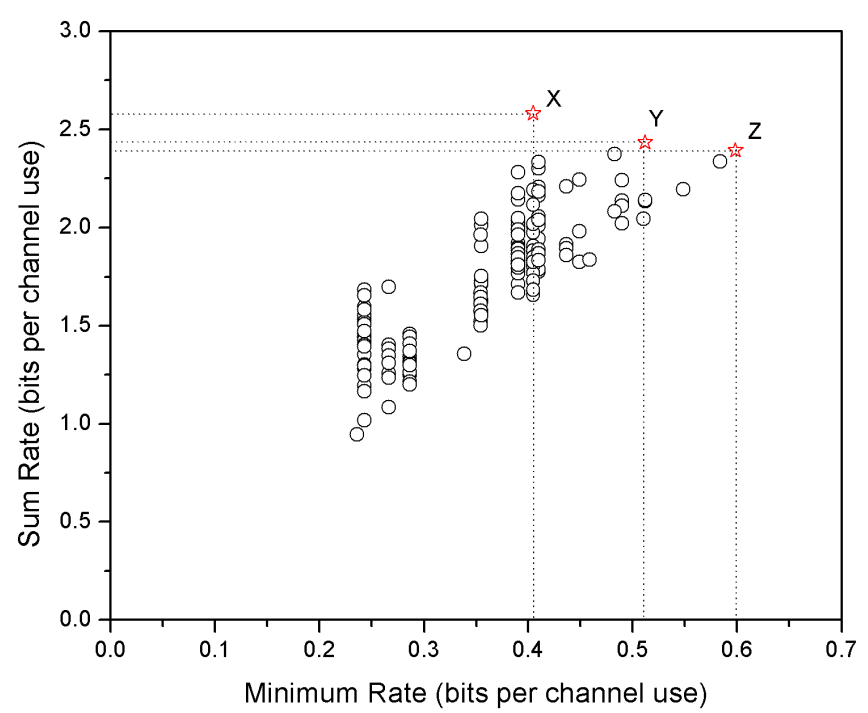

Fig. 1. Case study for a C\&F network with $L=4$ sources and $M=5$ relays, for a channel realization which leads to a solution set with Pareto frontier.

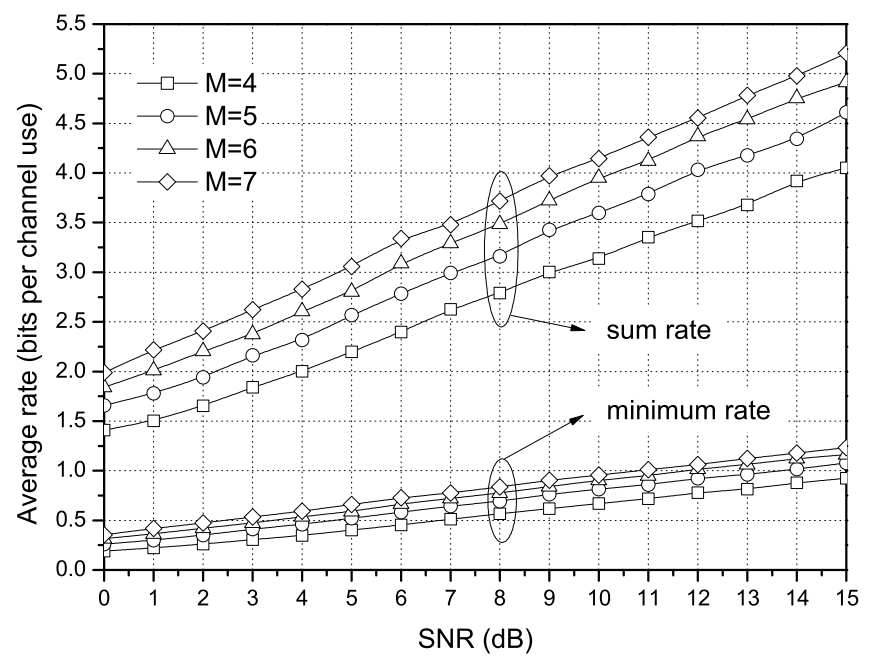

Fig. 2. Average Sum Rate and Average Minimum Rate after Algorithm 1, for $L=4$ and $M=4,5,6,7$, over Gaussian distributed channels.

for the minimum rate, the solution which is chosen as optimal corresponds to point $\mathrm{X}$, a minimum requirement of $\mathcal{R}_{m}^{r} \in(0.4047,0.5119]$ leads to the choice of point $\mathrm{Y}$, while for every other requirement below the maximum minimum rate, Algorithm 2 chooses point $\mathrm{Z}$.

\section{B. Simulation Results}

In the following, we present simulation results for the proposed algorithms, for Gaussian distributed channel coefficients, $\mathcal{N}(0,1)$.

In Fig. 2, we study the effect of relay selection under the criterion of minimum rate maximization, as described in Section II-C1. A network with $L=4$ sources is considered, while the results are presented for a coalition formation of 4 relays, out of a total of $M=4,5,6,7$ available relays. The algorithm used for these simulations is Algorithm 1, which 
optimizes the minimum transmission rate achieved over the network. Simulations show that relay selection significantly improves the network performance, both in terms of minimum transmission rate and sum rate. Moreover, when the number of available relays is increased from $M=4$ to $M=5$, the performance of the network in terms of minimum rate is increased up to $3 \mathrm{~dB}$, for $S N R=15 \mathrm{~dB}$. Similar improvement is illustrated for the sum rate. When the number of relays is further increased, e.g. for $M=7$, an improvement for up to 6 $\mathrm{dB}$ is observed. In Fig. 2, when extra available relays are added to the network, e.g. the number of available relays increases from $M=6$ to $M=7$, it is observed that the performance improvement due to relay selection becomes smaller.

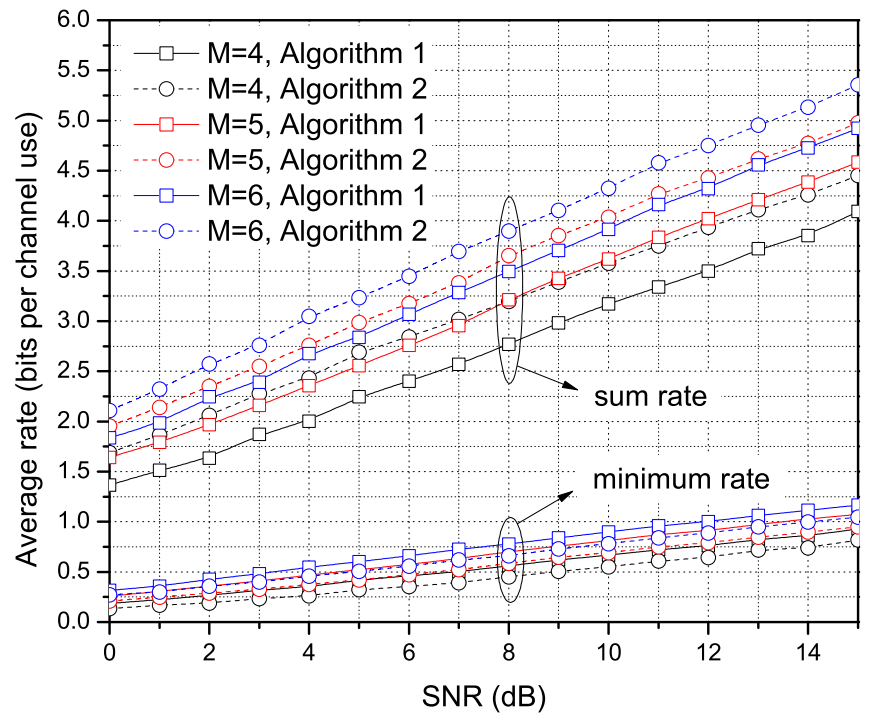

Fig. 3. Average Sum Rate and Average Minimum Rate before and after Algorithm 2, for $L=4$ and $M=4,5,6$, over Gaussian distributed channels.

On the other hand, in the results presented in Fig. 3, the minimum rate is not fixed to its maximum value, but it can be reduced, if this leads to a higher average sum rate. Algorithm 2 searches for the coding matrix $\mathbf{A}$ achieving the best sum rate, using all the candidate vectors in the vector sets of each relay. The presented results are for a network with $L=4$ sources and $M=4,5,6$ relays. For a cost in the average minimum transmission rate, a gain for up to $3 \mathrm{~dB}$ in the sum rate is achieved, for $M=4$. Similar gains are also observed, when the number $M$ of available relays increases. This leads to the conclusion that the sources can afford a reduction in their minimum rate requirements, in order to achieve higher network throughput. This is also fair among the sources, i.e. a source which accepts a lower rate for an adverse channel realization, will benefit from the increased sum rate for some other channel instance, since the benefits of this implementation are equally divided among the users.

Another interesting observation in Fig. 3 is the fact that the gain offered by the implementation of Algorithm 2 is the same or greater than the gain offered by the addition of an extra relay. This fact leads to important conclusion for practical design that an algorithmic optimization, which is performed by software, may be favorable, compared to adding an extra node to the network, leading to reduced overall cost. Furthermore, it is remarkable that, if both the optimization of Algorithm 2 and an increase of the number of relays from $M=4$ to $M=6$ is performed, a gain of $4-6 \mathrm{~dB}$ is illustrated, for the whole range of SNR values. Specifically, the sum rate achieved by $M=4$ relays, without optimization at $S N R=15 \mathrm{~dB}$, can be achieved by $M=6$ with optimization at $S N R=9 \mathrm{~dB}$, which corresponds to a reduction of the transmitted power by almost $75 \%$. Note also that the case of $M=6$ with optimization achieves higher minimum rate than the case of $M=4$ without optimization.

\section{Conclusions}

In this paper, the relay selection concept was introduced in order to maximize the sum and the minimum transmission rate in C\&F relaying networks. Moreover, an algorithmic approach was proposed in order to further optimize both rates, based on coalition formation game theory. The proposed method maximizes the sum rate, under specific requirements on the minimum rate and the transmitted power. It is remarkable that the algorithmic approach for the rates' optimization outperforms the addition of an extra relay. The simulation results also demonstrate that the proposed method achieves a well balanced tradeoff between sum and minimum rate, while it reduces considerably the energy consumption.

\section{REFERENCES}

[1] B. Nazer and M. Gastpar, "Compute-and-forward: Harnessing interference through structured codes," IEEE Trans. Inf. Theory, vol. 57, no. 10, pp. 6463-6486, Oct. 2011.

[2] U. Erez and R. Zamir, "Achieving $\frac{1}{2} \log (1+S N R)$ on the AWGN channel with lattice encoding and decoding," IEEE Trans. Inf. Theory, vol. 50, pp. 2293-2314, Oct. 2004.

[3] S. Zhang, S. Liew, and P. P. Lam, "Physical-layer network coding," in Proc. 2006 IEEE MobiComm, pp. 358-365.

[4] R. Ahlswede, N. Cai, S. R. Li, and R. W. Yeung, "Network information flow," IEEE Trans. Inf. Theory, vol. 46, no. 4, pp. 1204-1216, July 2000.

[5] M. Médard and A. Sprintson, Network coding: Fundamentals and applications, Academic Press, 2011.

[6] W. Saad, Z. Han, M. Debbah, A. Hjorungnes, and T. Basar, "Coalitional game theory for communication networks," IEEE Signal Processing Magazine, vol. 26, no. 5, pp. 77-97, Sep. 2009.

[7] C. Feng, D. Silva, and F. R. Kschischang, "Design criteria for lattice network coding," in Proc. IEEE 45th Annual Conference on Information Sciences and Systems (CISS), Baltimore, MD, USA, 2011.

[8] L. Wei and W. Chen, "Compute-and-forward network coding design over multi-source multi-relay channels," IEEE Trans. Wireless Commun., vol. 11, no. 9, pp. 3348-3357, Sep. 2012.

[9] A. Mejri and G.R.-B. Othman, "Practical Physical Layer Network Coding in Multi-Sources Relay Channels via the Compute-and-Forward," in Proc. IEEE Wireless Communications and Networking Conference Workshops (WCNCW 2013), pp.166,171, Shanghai, China, 7-10 April 2013.

[10] S.-N. Hong and G. Caire, "Antenna and user selection for a cloud base station with (Reverse) Compute and Forward," in Proc. IEEE Globecom Workshops, pp. 261-266, Anaheim, California, USA, 3-7 Dec. 2012.

[11] "C-RAN: the road towards green RAN," China Mobile Res. Inst., Beijing, China, Oct. 2011, White Paper, ver. 2.5.

[12] S. Banerjee, H. Konishi, and T. Sönmez, "Core in a simple coalition formation game," Social Choice and Welfare, vol. 18, no. 1, pp. 135153,2001

[13] U. Fincke and M. Pohst, "Improved Methods for Calculating Vectors of Short Length in a Lattice, Including a Complexity Analysis," Mathematics of Computation, vol. 44, no. 170, pp. 463471, 1985. 